\title{
Roles of self-assembly and beam damage in gas-assisted electron and ion beam induced processing.
}

\author{
Milos Toth $^{1}$ \\ 1. School of Physics and Advanced Materials, University of Technology, Sydney, \\ 15 Broadway, Ultimo, New South Wales 2007, Australia
}

Gas-assisted focused ion beam (FIB) processing enables high resolution, direct-write deposition and milling of a wide range of materials. Here I will review recent advances in FIB processing with an emphasis on:

- A new form of self-assembly in gas-assisted $\mathrm{Ga}^{+}$FIB-induced processing [1], and

- the use of oxygen ions for FIB milling of single crystal diamond [2].

Self-assembly can be used to overcome some of the limitations inherent to conventional, serial FIB methods in which a beam is scanned in a pattern that defines the nanostructure geometry. The limitations include throughput and the inability to fabricate complex 3D structures such as the Ga-filled, tapered $\mathrm{GaF}_{3}$ microcapillary shown in Figure 1. The structure was fabricated by irradiating GaN by a $\mathrm{Ga}^{+}$FIB that was scanned repeatedly over the rectangle seen in the figure, in the presence of a $\mathrm{XeF}_{2}$ precursor gas. Pillar growth proceeds through a self-ordering mechanism that emerges from an interplay between a number of physical and chemical mechanisms that include the generation of excess, mobile $\mathrm{Ga}$ atoms at the substrate surface, dissociation of $\mathrm{XeF}_{2}$ adsorbates by secondary electrons that impact the pillar sidewalls, sputtering, and self-masking caused by the tapered pillar geometry. The process can be used to grow multiple ordered or disordered pillars in parallel, and is fundamentally different from convention FIB-induced deposition and milling mechanisms [1].

A second significant limitation of conventional $\mathrm{Ga}^{+}$FIB processing techniques is damage and staining caused by the $\mathrm{Ga}^{+}$beam. This problem can, in some cases, be alleviated by the use of ions other than $\mathrm{Ga}^{+}$. For example, milling of single crystal diamond by an oxygen FIB yields a damage layer and (implanted oxygen) impurities which can be removed by post-processing methods that are less intrusive than those used to process diamond milled by $\mathrm{Ga}^{+}$ions [2]. The benefits and limitations of oxygen FIB milling of diamond will be discussed and compared to related electron beam processing techniques that enable minimally-invasive nanofabrication in the limit of negligible damage caused by momentum transfer and in the absence of staining caused by implantation. Specific examples of electron beam diamond processing techniques include damage-free etching using $\mathrm{H}_{2} \mathrm{O}$ as a precursor gas (Figure 2) [3], and chemical functionalization using $\mathrm{NF}_{3}$ as a precursor gas (Figure 3) [4].

\section{References:}

[1] Botman, A., Bahm, A., Randolph, S., Straw, M. \& Toth, M. Spontaneous Growth of Gallium-Filled Mi-crocapillaries on Ion-Bombarded GaN. Phys. Rev. Lett. 111, 135503 (2013).

[2] A. A. Martin, S. Randolph, A. Botman, M. Toth \& I. Aharonovich. Direct-write milling of diamond by a focused oxygen ion beam. Sci. Rep. (in press).

[3] Martin, A. A., Toth, M. \& Aharonovich, I. Subtractive 3D Printing of Optically Active Diamond Structures. Sci. Rep. 4, 5022 (2014).

[4] Shanley, T., Martin, A. A., Aharonovich, I. \& Toth, M. Localized chemical switching of the charge state of nitrogen-vacancy luminescence centers in diamond. Appl. Phys. Lett. 105, 063103 (2014). 

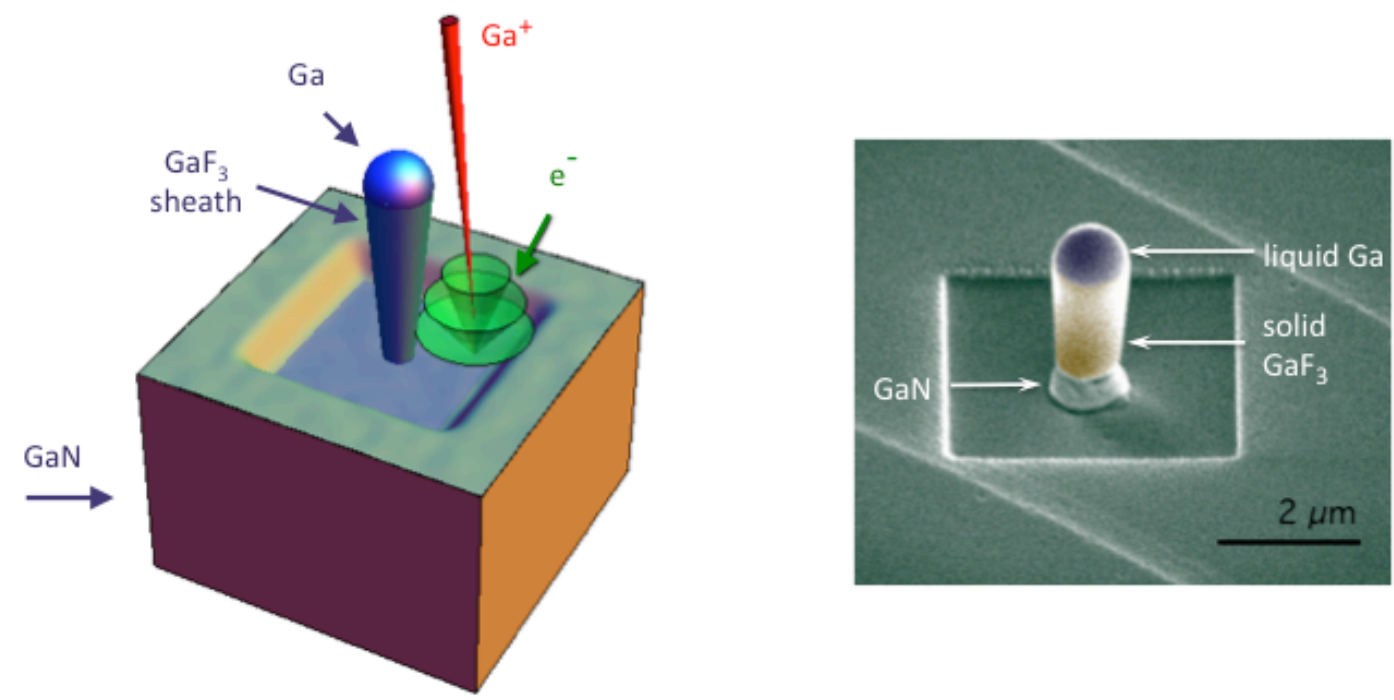

Figure 1. Schematic illustration (left) and a false-color SEM image (right) of a pillar grown by $\mathrm{Ga}^{+}$ion beam bombardment of $\mathrm{GaN}$ in an $\mathrm{XeF}_{2}$ environment. The pillar is comprised of a solid, tapered, hollow $\mathrm{GaF}_{3}$ sheath that is filled with liquid $\mathrm{Ga}[1]$.
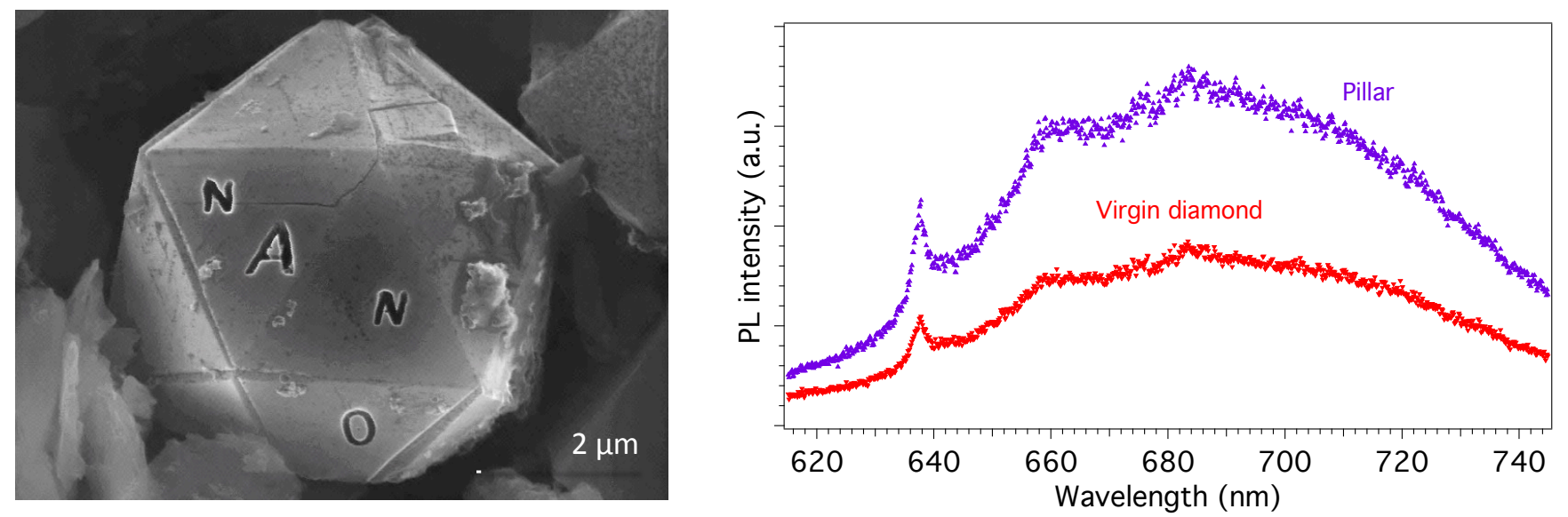

Figure 2. The letters "NANO" etched into a microdiamond using $\mathrm{H}_{2} \mathrm{O}$ as the etch precursor gas (left) and photoluminescence (PL) spectra showing PL enhancement by a diamond waveguide fabricated by electron beam induced etching [3].

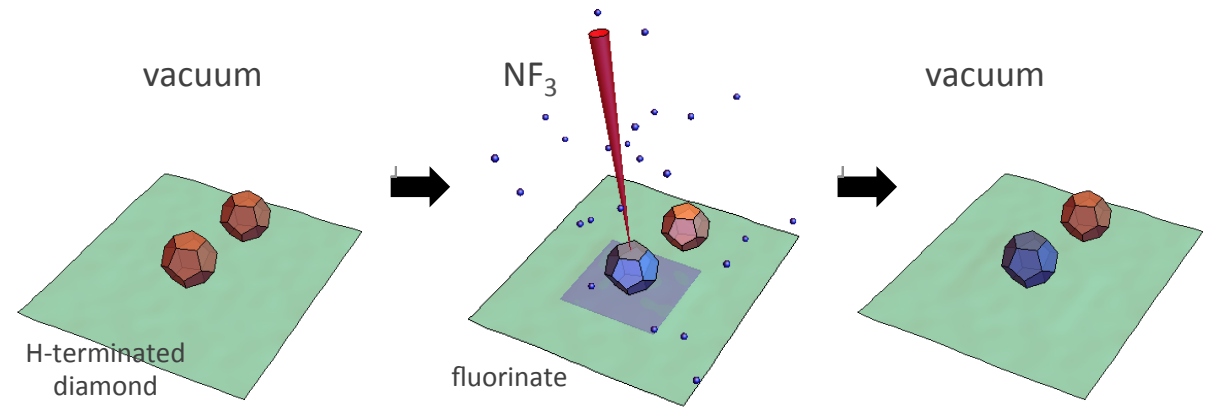

Figure 3. Schematic illustration of a direct-write process used to control the surface electronic structure and fluorescence properties of nanodiamonds by electron beam processing in a gaseous $\mathrm{NF}_{3}$ environment [4]. 